\title{
Abdominoperineal resection in the prone position: early outcomes at a tertiary institution in the Western Cape, South Africa
}

\author{
N Stevenson (D), AVV Lambrechts (D), T Forgan (i) \\ Division of Surgery, Faculty of Medicine and Health Sciences, Stellenbosch University and Tygerberg Hospital, South Africa
}

Corresponding author, email: nico.stev@gmail.com

Background: Extra-levator abdominoperineal resection (ELAPE) performed in the prone jack-knife position is a new technique in the developing world. Literature on the outcomes of ELAPE in a developing country context is scarce. The objective was to assess early outcomes after ELAPE in the prone jack-knife position, and to compare outcomes of patients who underwent the abdominal part of the procedure performed laparoscopically with an open group, at a tertiary institution in Cape Town.

Methods: Records of patients who underwent ELAPE for rectal adenocarcinoma from February 2011 to February 2017 at Tygerberg Hospital were retrospectively reviewed. Variables of interest included staging, rate of circumferential resection margin involvement (CRMI), intraoperative tumour perforation (IOP), perineal wound complications, early postoperative morbidity, length of intensive care unit (ICU) stay, duration of postoperative hospital stay and 30-day mortality rate.

Results: 52 patients (median age: 59 years) were included in the analysis. CRMI was evident in 16\% (8/49) of patients and IOP in 6\% (3/52). Perineal wound complications occurred in 32\% (16/50) of patients. Median length of ICU and postoperative hospital stay was 3 days and 7 days, respectively. Overall morbidity was 47\% (24/51) and the 30-day mortality rate was $3 \%(2 / 52)$. A significant difference in length of hospital stay was evident between the open and laparoscopic groups (11.5 days vs 6 days).

Conclusion: Prone abdominoperineal resection (APR), ELAPE, and laparoscopic ELAPE are acceptable and feasible procedures for patients with rectal cancer in the developing world, with outcomes being comparable to those determined in the developed world.

Keywords: rectal adenocarcinoma, extra-levator abdominoperineal section, prone abdominoperineal resection, laparoscopic extra-levator abdominoperineal resection

\section{Introduction}

Colorectal cancer (CRC) is less common in developing than developed countries, ${ }^{1}$ however, the incidence in developing countries is on the rise. ${ }^{2}$ While the incidence of CRC in developing countries is well documented, stage at presentation is less so. ${ }^{3}$ Limited available data indicates that patients in resource constrained environments present at an advanced stage of the disease. ${ }^{4}$ There is also evidence that the incidence of CRC is on the increase in younger patients ${ }^{5}$ and that they too present with more advanced stage tumours. ${ }^{6}$

Abdominoperineal resection/excision (APR/APE) is a surgical technique applied to cancers of the lower third of the rectum that are not amenable to sphincter preserving surgery. Since the description of the APR technique by Miles $^{7}$ in 1908, the technique has undergone modifications in an attempt to both improve oncological outcomes and to minimise perioperative complications. In addition to total mesorectal excision (TME), ${ }^{8}$ extra-levator APE (ELAPE) is one of the most significant modifications to the APR technique. ${ }^{9}$
ELAPE differs from conventional APR in that the mesorectum is not dissected off the levator muscles. Instead, the entire levator muscle is resected en bloc with the anal canal and lower rectum in order to decrease the surgical waist and to achieve a more cylindrical specimen. The aim is to decrease the rate of circumferential resection margin involvement (CRMI) and intraoperative tumour perforation (IOP). ${ }^{9}$ CRMI and IOP are known independent risk factors for tumour recurrence. ${ }^{10}$

The first ELAPE with the perineal phase of the procedure conducted in the prone position at Tygerberg Academic Hospital (TBH) was performed on 9 February 2011. Since then, this has become the preferred approach at our institution for patients with locally advanced rectal cancer involving the levator muscles or anus. Laparoscopy was first used to perform the abdominal phase of the procedure on 11 August 2011. It is therefore important to assess the outcomes of this recently adopted surgical technique that is utilised on a sizeable proportion of our patients with low rectal cancer. We present a large series of open and laparoscopic ELAPE/ prone APR from the developing world. 


\section{Aim of the study}

The aim of the study was to assess early outcomes after ELAPE in the prone jack-knife position at a tertiary institution in Cape Town, South Africa. A subgroup analysis was done comparing the group of patients who had the abdominal part of procedure performed laparoscopically with the open group. Primary endpoints included the rate of circumferential resection margin involvement (CRMI) and intraoperative tumour perforation (IOP). Secondary endpoints included stage at presentation, perineal wound complications, length of intensive care unit (ICU) stay, duration of postoperative hospital stay and 30-day mortality.

\section{Methods}

The records of adult patients (newly diagnosed rectal adenocarcinoma as well as recurrent rectal cancer) who underwent APR in the prone position/ELAPE, from February 2011 to February 2017, were reviewed retrospectively. Patients who had ELAPE were identified using a surgical gastroenterology database. Patients who underwent an ELAPE for anal squamous cell cancer and pelvic exenterations were excluded from the study.

Computed tomography (CT) of the chest and abdomen and a pelvic magnetic resonance imaging (MRI) formed part of the preoperative staging. The American Joint Committee on Cancer (AJCC) TNM (tumour, node, metastasis) staging system for colon and rectal cancer (7th edition) was used to determine tumour staging. A baseline carcinoembryonic antigen (CEA) was done for postoperative surveillance. A diverting sigmoid colostomy was performed on rectal cancer patients prior to the commencement of neoadjuvant therapy, if a colonoscope or sigmoidoscope could not be advanced past the tumour. Each patient's management pathway was decided at a combined surgical oncology multidisciplinary meeting.

Neoadjuvant chemo-radiation (CRT) was the standard of care for locally advanced rectal cancer (T3/4, lymph-node positive or threatened circumferential resection margins (CRMs). The neoadjuvant regime comprised long course chemoradiotherapy. For those patients who were downstaged following neoadjuvant CRT, the decision to administer adjuvant chemotherapy was individualised. Patients who did not qualify for chemotherapy as a result of ischemic heart disease or other contraindications to chemotherapy received short course neoadjuvant radiotherapy. All patients had an MRI six weeks post completion of neoadjuvant CRT to assess their response to treatment. Surgery was performed six to twelve weeks after neoadjuvant treatment.

Patients' physical status was categorised according to the American Society of Anesthesiologists physical status classification system (ASA PS). ${ }^{10}$

Statistical analysis was performed using SPSS for Windows version 25.0 (SPSS Inc., Chicago, IL). Descriptive statistics were computed for demographic and clinical data and presented as medians and means, where applicable, with associated ranges. Pearson's chi-square test or Fisher's exact test were used, where appropriate, to determine the significance of differences in proportions of categorical data. Statistical significance was set at $p<0.05$.

Table I: Patient demographics and preoperative clinical data

\begin{tabular}{|c|c|c|c|c|c|c|c|c|}
\hline \multirow[t]{2}{*}{ Variables } & & \multicolumn{6}{|c|}{ Patient groups } & \multirow[b]{2}{*}{$p$-value } \\
\hline & & \multicolumn{2}{|c|}{$\begin{array}{l}\text { Laparoscopic } \\
\quad(n=35)\end{array}$} & \multicolumn{2}{|c|}{$\begin{array}{l}\text { Open } \\
(n=17)\end{array}$} & \multicolumn{2}{|c|}{$\begin{array}{l}\text { Total } \\
(n=52)\end{array}$} & \\
\hline \multicolumn{2}{|l|}{ Age in years [median (range)] } & \multicolumn{2}{|c|}{$59(29-84)$} & \multicolumn{2}{|c|}{$59(41-75)$} & \multicolumn{2}{|c|}{$59(29-84)$} & $>0.05$ \\
\hline \multicolumn{2}{|l|}{ ASA PS [median (range)] } & \multicolumn{2}{|c|}{$2(1-3)$} & \multicolumn{2}{|c|}{$2(1-3)$} & \multicolumn{2}{|c|}{$2(1-3)$} & \\
\hline & & $\mathbf{N}$ & $\%$ & $\mathbf{N}$ & $\%$ & $\mathbf{N}$ & $\%$ & \\
\hline \multirow[t]{3}{*}{ Gender } & & & & & & & & $<0.05$ \\
\hline & Male & 19 & 54 & 14 & 82 & 33 & 63 & \\
\hline & Female & 16 & 46 & 3 & 18 & 19 & 37 & \\
\hline \multicolumn{9}{|l|}{ Raised preoperative CEA } \\
\hline & Yes & 13 & 37 & 7 & 41 & 20 & 38 & \\
\hline & No & 22 & 63 & 10 & 59 & 32 & 62 & \\
\hline \multicolumn{9}{|l|}{ Preoperative blood transfusion } \\
\hline & Yes & 1 & 2 & 0 & 0 & 1 & 2 & \\
\hline & No & 34 & 98 & 17 & 100 & 51 & 97 & \\
\hline \multicolumn{9}{|l|}{ Preoperative diverting colostomy } \\
\hline & Yes & 17 & 48 & 7 & 41 & 24 & 46 & \\
\hline & No & 18 & 52 & 10 & 59 & 28 & 54 & \\
\hline \multicolumn{9}{|l|}{ Recurrent rectal cancer } \\
\hline Low anterior resection & & 2 & 6 & 1 & 6 & 3 & 6 & \\
\hline $\begin{array}{l}\text { Total colectomy \& end } \\
\text { ileostomy }\end{array}$ & & 0 & 0 & 1 & 6 & 1 & 2 & \\
\hline \multicolumn{2}{|c|}{$\begin{array}{l}\text { Tumour distance from anal verge in } \mathrm{cm} \\
\text { [median (range)] }\end{array}$} & \multicolumn{2}{|c|}{$0(0-5)$} & \multicolumn{2}{|c|}{$3(0-5)$} & \multicolumn{2}{|c|}{$2(0-5)$} & \\
\hline
\end{tabular}

CEA - carcinoembryonic antogen, ASA PS - American Society of Anesthesiologists physical status classification 


\section{Results}

\section{Patient demographic and preoperative clinical data}

A total of 52 patients were treated with prone APR between February 2011 and February 2017. The median age of the sample overall was 59 years (range: 29-84 years) (see Table I). No significant difference in age between the open and laparoscopic group was evident $(p>0.05)$. Almost two-thirds of the sample overall were male $(63 \%, 33 / 52)$, and significantly more males underwent the open vs the laparoscopic procedure $(82 \%$ vs $54 \%, p<0.05)$. Over a third of the total sample (38\%) had a raised preoperative CEA. The median ASA classification was 2. Only 3\% of patients had a preoperative blood transfusion. Of the total sample, 24 patients $(46 \%)$ had a preoperative diverting sigmoid colostomy done prior to the commencement of neoadjuvant radiotherapy.

Three patients $(6 \%)$ had an APR after rectal cancer recurrence and one patient $(2 \%)$ developed rectal cancer after previous surgery for colon cancer. The three patients with recurrent rectal cancer previously underwent low anterior resection. The patient who had colon cancer had previously undergone a total colectomy and end ileostomy which was performed at a different institution. The median tumour height above the anal verge in the sample overall was $2 \mathrm{~cm}$ (range: $0-5 \mathrm{~cm}$ ).

\section{Neoadjuvant and adjuvant therapy}

Thirty-seven patients (71\%) received neoadjuvant chemoradiotherapy (CRT). Six patients $(12 \%)$ received only neoadjuvant short course radiotherapy (SCRT). Nine patients $(17 \%)$ did not receive any neoadjuvant therapy. The patients who did not receive neoadjuvant therapy had either a contraindication to neoadjuvant therapy, had already received neoadjuvant therapy previously (in the case of rectal cancer recurrence), or had early stage tumours that did not justify the use of neoadjuvant therapy. The percentage of patients who received neoadjuvant CRT and neoadjuvant SCRT was comparable between the laparoscopic and open groups $(p=0.225)$.

Adjuvant chemotherapy was given in 22 patients (42\%) whereas $24(46 \%)$ received no adjuvant therapy. The percentage of patients who received adjuvant chemotherapy in the open and the laparoscopic groups was comparable $(p=0.619)$. Data on adjuvant therapy was missing in six patients $(12 \%)$.

\section{Clinical staging}

Most patients in the cohort had locally advanced rectal cancer on initial staging MRI, with either T3 (42\%) or T4 (44\%) lesions evident (see Table II). Overall, 16 patients $(30 \%)$ were diagnosed as having stage 2 rectal cancer and 31 patients $(60 \%)$ were diagnosed as having stage 3 after

Table II: Clinical staging pre and post neoadjuvant therapy

\begin{tabular}{|c|c|c|c|c|c|c|c|}
\hline \multirow{3}{*}{ Variables } & \multicolumn{4}{|c|}{ Abdominal component } & & & \multirow{3}{*}{$p$-value } \\
\hline & \multicolumn{2}{|c|}{ Laparoscopic $(n=35)$} & \multicolumn{2}{|c|}{ Open $(n=17)$} & \multicolumn{2}{|c|}{ Total $(n=52)$} & \\
\hline & $N$ & $\%$ & $N$ & $\%$ & $N$ & $\%$ & \\
\hline \multicolumn{7}{|l|}{ Clinical (MRI) T-stage } & $>0.05$ \\
\hline $\mathrm{cT} 1$ & 0 & 0 & 0 & 0 & 0 & 0 & \\
\hline $\mathrm{cT} 2$ & 3 & 9 & 2 & 12 & 5 & 10 & \\
\hline cT3 & 15 & 43 & 7 & 41 & 22 & 42 & \\
\hline cT4 & 15 & 43 & 8 & 47 & 23 & 44 & \\
\hline No preoperative MRI & 2 & 5 & 0 & 0 & 2 & 4 & \\
\hline \multicolumn{7}{|l|}{ Clinical (MRI) N-stage } & $>0.05$ \\
\hline $\mathrm{cNO}$ & 10 & 29 & 8 & 47 & 18 & 34 & \\
\hline $\mathrm{cN} 1$ & 6 & 17 & 2 & 12 & 8 & 15 & \\
\hline $\mathrm{cN} 2$ & 17 & 49 & 7 & 41 & 24 & 48 & \\
\hline No preoperative MRI & 2 & 5 & 0 & 0 & 2 & 3 & \\
\hline \multicolumn{7}{|l|}{ Clinical TNM stage } & $>0.05$ \\
\hline Stage 1 & 2 & 6 & 1 & 6 & 3 & 6 & \\
\hline Stage 2 & 8 & 23 & 8 & 47 & 16 & 30 & \\
\hline Stage 3 & 23 & 65 & 8 & 47 & 31 & 60 & \\
\hline Stage 4 & 0 & 0 & 0 & 0 & 0 & 0 & \\
\hline No MRI & 2 & 6 & 0 & 0 & 2 & 4 & \\
\hline \multicolumn{7}{|c|}{ Clinical TNM stage post neoadjuvant therapy } & $>0.05$ \\
\hline Stage 1 & 0 & 0 & 0 & 0 & 0 & 0 & \\
\hline Stage 2 & 13 & 37 & 7 & 41 & 20 & 38 & \\
\hline Stage 3 & 15 & 43 & 5 & 30 & 20 & 38 & \\
\hline Stage 4 & 0 & 0 & 0 & 0 & 0 & 0 & \\
\hline No MRI & 7 & 20 & 5 & 29 & 12 & 24 & \\
\hline
\end{tabular}


initial clinical staging. No significant differences were evident between the open and laparoscopic groups in terms of clinical MRI T-stage, clinical MRI N-stage, or clinical TNM stage $(p>0.05)$.

\section{Early outcomes after ELAPE}

The CRM was involved (tumour $\leq 1 \mathrm{~mm}$ of CRM) in eight patients $(16 \%)$ overall (see Table III). No significant difference in CRMI was evident between the laparoscopic and open groups $(p>0.05)$. Five of the patients with CRMI were male $(62 \%)$ and three were female $(38 \%)$.

IOP occurred in three $(6 \%)$ patients overall. No significant difference in rate of IOP was evident between the laparoscopic and open groups $(p>0.05)$.

Perineal wound complications occurred in a third (32\%) of the sample. Complications included perineal wound sepsis and wound dehiscence. No significant difference in the number of perineal wound complications was evident between the laparoscopic and open groups $(p>0.05)$. No statistically significant association between neoadjuvant CRT and perineal wound complications was found ( $p=$ 0.535).

A total of twenty-four patients (47\%) had some form of postoperative morbidity. No significant difference in rate of postoperative morbidity was evident between the laparoscopic and open groups $(p>0.05)$.

The median length of ICU/High Care stay was three days (range: 0-12 days). No significant difference in length of ICU stay was evident between the laparoscopic and open groups $(p>0.05)$. The median length of hospital stay was seven days (range: $3-55$ days). The open group demonstrated a significantly longer hospital stay than the laparoscopic group (11.5 days vs 6 days).

A 30-day mortality rate of 3\% (2/52) was noted, with two patients in the open group passing away within 30 days of surgery. One patient passed away two days after surgery in ICU. This patient's post-mortem examination was inconclusive as to the cause of death. The second patient, after being discharged from the hospital in a stable condition on day ten postoperatively, was found deceased in his bed by his family 24 days post-surgery. The exact cause of death could not be determined as the attending physician did not request a post-mortem examination.

\section{Other early postoperative complications}

A low rate of $4 \%$ or less for other postoperative complications was noted in the sample overall. These included postoperative ileus $(2 / 52,4 \%)$, iatrogenic urethral injury $(2 / 52,4 \%)$, stomal prolapse $(1 / 52,2 \%)$, pelvic collection $(1 / 52,2 \%)$, sacral osteomyelitis $(1 / 52,2 \%)$, and pulmonary embolism $(1 / 52,2 \%)$.

\section{Discussion}

The current study described the early outcomes after ELAPE/prone APR in 52 patients (those newly diagnosed with rectal adenocarcinoma and those with recurrent rectal cancer) operated on at a tertiary hospital in South Africa and compared early outcomes in patients in which the open and laparoscopic techniques were performed.

The patient population reported on in this study was relatively small compared to studies conducted in developed countries, yet comparable with those studies that have reported on APR in developing countries. ${ }^{3}$ The relatively small sample may reflect the low incidence of rectal cancer in developing countries. ${ }^{11}$ In a retrospective descriptive study conducted at a tertiary institution in Nigeria, only 36 patients underwent APR over an 18-year period. ${ }^{3}$ A recent South African comparative study of ELAPE and conventional APR (cAPR) conducted over a four-year period included a total of 56 patients, 26 of whom had an ELAPE. ${ }^{12}$

The median age of patients in this study was 59 years, which is higher than that determined in rectal cancer patients in series from other developing countries ${ }^{3,12}$ and lower than the age of rectal cancer patients reported on in series originating from developed countries. ${ }^{13}$ These differences in age may be explained by proportional differences in racial distribution in developing countries where life expectancy is lower, ${ }^{14}$ and where earlier onset of rectal cancer is evident among ethnic groups other than Caucasian. ${ }^{15}$

We determined a larger proportion of males than females in the sample overall and in the open group compared to the laparoscopic group. Previous findings have suggested that males have a higher risk of CRMI due to the anatomy of the male pelvis making dissection more difficult, yet other studies have shown the opposite. ${ }^{16}$

A substantial proportion of patients in this study had locally advanced cancer on initial staging MRI, with either a $\mathrm{T} 3$ or T4 tumour, as well as an advanced $\mathrm{N}$-stage. In addition, almost half the patients had N2 disease. A conclusion about

Table III: Outcomes of laparoscopic, open and total patient groups

\begin{tabular}{|c|c|c|c|c|c|c|c|}
\hline \multirow{3}{*}{ Variables } & \multicolumn{4}{|c|}{ Patient groups } & & & \multirow{3}{*}{$p$-value } \\
\hline & \multicolumn{2}{|c|}{$\begin{array}{l}\text { Laparoscopic } \\
\quad(n=35)\end{array}$} & \multicolumn{2}{|c|}{$\begin{array}{c}\text { Open } \\
(n=17)\end{array}$} & \multicolumn{2}{|c|}{$\begin{array}{c}\text { Total } \\
(n=52)\end{array}$} & \\
\hline & $N$ & $\%$ & $N$ & $\%$ & $N$ & $\%$ & \\
\hline CRMI & $3 / 33$ & 9 & $5 / 16$ & 31 & $8 / 49$ & 16 & $>0.05$ \\
\hline IOP & 2 & 6 & 1 & 6 & 3 & 6 & $>0.05$ \\
\hline Perineal wound complications & $10 / 34$ & 29 & $6 / 16$ & 38 & $16 / 50$ & 32 & $>0.05$ \\
\hline Morbidity & $14 / 34$ & 41 & 10 & 58 & $24 / 51$ & 47 & $>0.05$ \\
\hline 30-day mortality & 0 & 0 & 2 & 12 & 2 & 3 & \\
\hline Length of ICU stay in days [median (range)] & \multicolumn{2}{|c|}{$3(1-2)$} & \multicolumn{2}{|c|}{$4(0-7)$} & \multicolumn{2}{|c|}{$3(0-12)$} & $>0.05$ \\
\hline Length of hospital stay in days [median (range)] & \multicolumn{2}{|c|}{$6(3-32)$} & \multicolumn{2}{|c|}{$11.5(4-55)$} & \multicolumn{2}{|c|}{$7(3-55)$} & $<0.05$ \\
\hline
\end{tabular}

CRMI - circumferential resection margin involvement, IOP - intraoperative tumour perforation 
stage at presentation cannot be reached from the current data as the patients included in the study were selected according to surgical procedure and not rectal cancer status. The patients in this cohort were treated at a particular academic referral hospital, which may not reflect the population in the Western Cape, or in South Africa as a whole.

The CRMI and IOP rate of $16 \%$ and $6 \%$ respectively determined in this study is acceptable when compared to rates achieved in the developed world. ${ }^{17}$ The current study did not compare the ELAPE group with a cAPE group due to the historical nature of patients who had cAPE at TBH. Changes in neoadjuvant treatment make the comparison of these two groups unreliable. Whether the rates of CRMI and IOP translate to acceptable oncological outcomes, i.e. locoregional recurrence and disease-free survival, remains to be confirmed by longer-term follow-up of the current population. The difference in CRMI rates between the open and the laparoscopic groups, although not statistically significant, may be due to the larger proportion of T3 and higher-grade tumours evident in the open group.

Perineal wound complications were a frequent occurrence across the two groups in this study, with $32 \%$ of patients overall developing some form of perineal wound morbidity, including wound sepsis or wound dehiscence. No significant difference in the number of occurrences of perineal wound complications was evident between the laparoscopic and open groups, which is to be expected given that the perineal phase of the dissection was performed using the same technique (i.e. prone position) in both groups. Although a perineal wound complication rate of $32 \%$ appears high, it is lower than that determined by Musters et al. ${ }^{18}$ in a metaanalysis of perineal wound healing after abdominoperineal resection for rectal cancer $(37.6 \%)$.

No significant difference in length of ICU stay between the two groups was determined; however, we found that patients in the laparoscopic group had a significantly shorter length of hospital stay than those in the open group. In a healthcare facility such as $\mathrm{TBH}$, characterised by an overwhelming patient load and high demand on bed availability, this an important finding. Although many series conducted in developed countries have supported this finding, ${ }^{19}$ some have found no difference in length of hospital stay between open and laparoscopic ELAPE. ${ }^{20}$

We believe that the laparoscopic approach is ideally suited for the abdominal phase of ELAPE as dissection during the abdominal phase is usually halted before the level of the tumour has been reached and the local oncological resection is mainly done during the perineal phase.

\section{Study limitations}

Limitations of the present study include the retrospective collection of data, which may have affected both the quality and reliability of the data. In addition, the data were collected from a single tertiary academic centre, with most surgeries being performed by a single surgeon. Given the aforementioned, our outcomes may not reflect those in other units or those by surgeons in the developing world when using the same technique. Although our oncological and perioperative outcomes compare favourably with those from the developed world, owing to the historical nature of cAPE at our institution, we did not have a control group for comparison. Selection bias may have occurred in this study where patient and tumour factors determined the type of procedure. Therefore, we cannot confirm that the laparoscopic approach is superior to the open approach, but that the laparoscopic approach is feasible and safe. Despite the small sample size, a strength of this study is that it is the largest reported consecutive series on ELAPE from the developing world, and the first to include a subgroup of laparoscopic versus open ELAPE patients.

\section{Conclusion}

This study assessed the early outcomes of a sample of rectal cancer patients in the Western Cape, South Africa, after ELAPE with the perineal dissection performed in the prone position. In terms of early outcomes, satisfactory results were determined when compared to those from the developed world. Our findings suggest that laparoscopic ELAPE can achieve CRMI and IOP rates that are equivalent to those of open ELAPE, with the added advantage of a significantly shorter hospital stay. The findings from this study suggest that ELAPE and laparoscopic ELAPE are both acceptable and feasible when applied to patients with rectal cancer in the developing world.

Recommendations for future research include the longterm follow-up of the current sample to assess the long-term oncological outcomes of ELAPE and laparoscopic ELAPE in the developing world.

\section{Conflict of interest}

The authors declare no conflict of interest.

\section{Funding source}

No funding source to be declared.

\section{Ethical approval}

The study was approved by the Health Research Ethics Committee (HREC) of Stellenbosch University (Reference: S15/03/066).

\section{ORCID}

N Stevenson (iD https://orcid.org/0000-0002-0755-9806 AVV Lambrechts (iD https://orcid.org/0000-0003-0565-3978 T Forgan (iD https://orcid/org/0000-0001-6764-6699

\section{REFERENCES}

1. Haggar FA, Boushey RP. Colorectal cancer epidemiology: incidence, mortality, survival, and risk factors. Clin Colon Rectal Surg. 2009;6(212):191-7. https://doi. org/10.1055/s-0029-1242458.

2. Bishehsari F, Mahdavinia M, Vacca M, Malekzadeh R, Mariani-Costantini R. Epidemiological transition of colorectal cancer in developing countries: Environmental factors, molecular pathways, and opportunities for prevention. World J Gastroenterol. 2014;20(20):6055-72. https://doi. org/10.3748/wjg.v20.i20.6055.

3. Alatise OI, Lawal OO, Adesunkanmi AK, Osasan SA Surgical outcome of abdominoperineal resection for low rectal cancer in a Nigerian tertiary institution. World J Surg. 2009;33(2):233-9. https://doi.org/10.1007/s00268-008-98170.

4. Patil PS, Saklani A, Gambhire P, et al. Colorectal cancer in India: an audit from a tertiary center in a low prevalence 
area. Indian J Surg Oncol. 2017;8(4):484-90. https://doi. org/10.1007/s13193-017-0655-0.

5. Mckay A, Donaleshen J, Helewa RM, et al. Does young age influence the prognosis of colorectal cancer: a populationbased analysis. 2014:1-10. https://doi.org/10.1186/14777819-12-370.

6. Dozois EJ, Boardman LA, Suwanthanma W, et al. Youngonset colorectal cancer in patients with no known genetic predisposition: can we increase early recognition and improve outcome? Medicine (Baltimore). 2008;87(5):259-63. https:// doi.org/10.1097/MD.0b013e3181881354.

7. Miles WE. A method of performing abdomino-perineal excision for carcinoma of the rectum and of the terminal portion of the pelvic colon. CA Cancer J Clin. 1971;21(6):3614. https://doi.org/10.3322/canjclin.21.6.361.

8. Heald RJ, Husband EM, Ryall RDH. The mesorectum in rectal cancer surgery - the clue to pelvic recurrence? Br J Surg. 1982;69(10):613-6. https://doi.org/10.1002/bjs.1800691019.

9. Holm T, Ljung A, Häggmark $T$, Jurell $G$, Lagergren $J$. Extended abdominoperineal resection with gluteus maximus flap reconstruction of the pelvic floor for rectal cancer. Br J Surg. 2007;94(2):232-8. https://doi.org/10.1002/bjs.5489.

10. Anderin C, Granath F, Martling A, Holm T. Local recurrence after prone vs supine abdominoperineal excision for low rectal cancer. Color Dis. 2013;15(7):812-5. https://doi.org/10.1111/ codi. 12148.

11. Fitz-henry J. Peri-operative care series. Ann R Coll Surg Engl 2011;93:185-7. https://doi.org/10.1308/147870811X56 5070 .

12. Oostenbroek M, Coetzee E, Boutall A, Baigrie R, Goldberg P. Comparative study of extralevator vs. conventional abdominoperineal excision in a single centre in the developing world. South African J Surg. 2016;54(4):34-9.

13. West NP, Anderin C, Smith KJE, Holm T, Quirke P. Multicentre experience with extralevator abdominoperineal excision for low rectal cancer. Br J Surg. 2010;97(4):588-99. https://doi.org/10.1002/bjs.6916.

14. Statistics South Africa. Census 2011: population dynamics in South Africa; 2012.

15. Elliot MS, Steven DM, Terblanche J. Abdominoperineal resection of the rectum for carcinoma at Groote Schuur Hospital, Cape Town, 1971-1982. S Afr Med J. 1984;65(11):411-3.

16. Klein M, Fischer A, Rosenberg J, Gögenur I. Extralevatory abdominoperineal excision (ELAPE) does not result in reduced rate of tumor perforation or rate of positive circumferential resection margin: a nationwide database Study. Ann Surg. 2015;261(5):933-8. https://doi.org/10.1097/ SLA.0000000000000910.

17. Krishna A, Rickard MJFX, Keshava A, Dent OF, Chapuis PH. A comparison of published rates of resection margin involvement and intra-operative perforation between standard and "cylindrical" abdominoperineal excision for low rectal cancer. Color Dis. 2013;15(1):57-65. https://doi.org/10.1111/ j.1463-1318.2012.03167.x.

18. Musters GD, Buskens CJ, Bemelman WA, Tanis PJ. Perineal woundhealingafterabdominoperinealresectionforrectalcancer: a systematic review and meta-analysis. Dis Colon Rectum. 2014;57(9):1129-39. https://doi.org/10.1097/DCR.00000000 00000182 .

19. Zhang F-W, Zhou Z-Y, Wang H-L, et al. Laparoscopic versus open surgery for rectal cancer: a systematic review and metaanalysis of randomized controlled trials. Asian Pac J Cancer Prev. 2014;15(22):9985-96.

20. Ahmad NZ, Racheva G, Elmusharaf H. A systematic review and meta-analysis of randomized and non-randomized studies comparing laparoscopic and open abdominoperineal resection for rectal cancer. Color Dis. 2013;15(3):269-77. https://doi. org/10.1111/codi.12007. 